





\title{
Exhibition of Paintings and
} Drawings Showing the Later Tendencies in Art

\author{
April 16 to May 15
}

The Pennsylvania Academy of the Fine Arts

\section{PHILADELPHIA}



Paintings and Drawings by American Artists showing the Later

\section{Tendencies in Art}

An Exhibition held by courtesy of the Pennsylvania Academy of the Fine Arts, "Broad and Cherry Streets, Philądelphia.

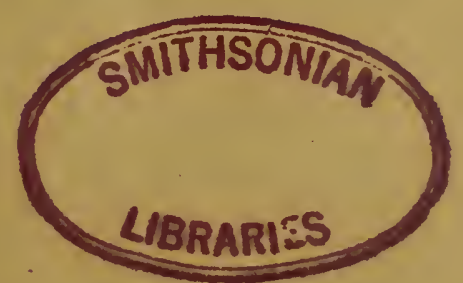




\section{COMMITTEE OF SELECTION}

Thomas Benton

Paul Burlin

Arthur B. Carles
Bernard Gussow

Joseph Stella

Alfred Stieglitz

\section{William Yarrow}

HANGING COMMITTEE

Paul Burlin

Thomas Benton
Arthur B. Carles

Alfred Stieglitz

William Yarrow 


\section{CATALOGUE OF THE EXHIBITION \\ GALLERY I.}

FLORINE STETTHEIMER.

I A Day in West Point.

C. BERTRAM HARTMAN.

2 The Crown.

E. B. GROSSMAN.

3 Helen by the Sea.

J. TORRES-GARCIA.

4 Spanish Town.

JOSEPH M. GARVEY.

5 The Dance.

D. W. MCCOUCH.

6 Landscape, with Church.

B. J. O. NORDFELDT.

7 Corn Dance: San Ildefonso.

ALLEN TUCKER.

8 Scherzo: A Song. 
EDWARD FISK.

9 Adirondack Lake.

PRESTON DICKINSON.

to House Forms and Landscape.

JOHN PANDICK.

II Portrait.

SAMUEL HALPERT.

I2 Interior.

JAMES H. DAUGHERTY.

I3 Still Life.

JOHN PANDICK.

I4 Composition.

EDWARD FISK.

I5 Still Life.

GEORGE BIDDLE.

I6 Allegro.

EMILE BRANCHARD.

I7 The Lake.

HUGO ROBUS.

I8 Fire Escapes and Things.

EDWARD NAGLE.

I9 Forty-second Street, Going West. 
PAINTINGS AND DRAWINGS.

GEORGE BIDDLE.

20 Il Penseroso.

EMILE BRANCHARD.

2 The Grove.

JAMES H. DAUGHERTY.

22 Crossing the Plains.

GEORGE F. OF.

23 Still Life.

J. TORRES-GARCIA.

24 Business Town.

RAFAEL SALA.

25 On the Mediterranean Seaboard.

MORRIS KANTOR.

26 Sunset.

RICHARD BOIX.

27 Crash.

WALTER PACH.

28 The Waterfall.

FLORINE STETTHEIMER.

29 Asbury Park, South.

RICHARD BOIX.

30 Three. 


\section{PAINTINGS AND DRAWINGS.}

E. E. CUMMINGS.

3I Noise: Number Five.

GEORGE BIDDLE.

32 Flute Player and Ballad Singer.

KATHERINE L. DREIER.

33 Bibi.

ELIZABETH NAGEL.

34 Pastel.

JOSEPH STELLA.

35 Water Lilies.

HAMILTON EASTER FIELD.

36 Colonial.

ARNOLD FRIEDMAN.

37 Pressure.

ELIZABETH NAGEL.

${ }^{8}$ Painting. 


\section{NORTH CORRIDOR.}

BEN BENN.

39 Still Life.

HENRY FITCH TAYLOR.

40 Abstraction.

A. S. BAYLINSON.

4I Figure.

MORRIS KANTOR.

42 Quiet Waters.

ARNOLD FRIEDMAN.

43 Flight.

J. TORRES-GARCIA.

44 Fourteenth Street.

WM. ZORACH.

45 The Waterfall.

BERNARD GUSSOW.

46 The Woods.

HUGO ROBUS.

47 Promenade. 
GEORGE BIDDLE.

48 Nude.

CARL NEWMAN.

49 Painting: Number One.

50 Painting: Number Three.

5 I Toys.

HUNT DIEDERICH.

52 Polo: Silhouette.

MAURICE STERNE.

53 The Circus.

YASUO KUNIYOSHI.

54 Fishermen's Huts.

HUNT DIEDERICH.

55 Polo: Silhouette.

LAYMAN SAYEN.

$5^{6}$ Landscape.

B. J. O. NORDFELDT.

57 Father and Son.

LYMAN SAYEN.

${ }^{8}$ Landscape.

WOOD GAYLOR.

59 Vampires.

Lent by Mrs. M. Erdrich. 
A. S. BAYLINSON.

6o Figure.

HENRY FITCH TAYLOR.

6I Ave Maria.

E. E. CUMMINGS.

62 Noise: Number One.

ELIZABETH NAGEL.

63 Drawing.

EMILE BRANCHARD.

64 High Tide.

65 To the Hills.

66 Rocky Shore.

ALLEN TUCKER.

67 Portrait in Dull Red. 


\section{GALLERY $\mathbf{H}$.}

W. GLACKENS.

68 Finnish Woman.

RAFAEL SALA.

69 Still Life.

W. GLACKENS.

70 Avery's Point.

ROBERT LAURENT.

7 I Rocks and Boat.

A. H. MAURER.

72 Head: Number Two.

NAN WATSON.

73 Still Life.

Lent by Mrs. Willard Force.

GUS MAGER.

74 Wyoming, New Jersey: Landscape.

ROBERT LAURENT.

75 Rocks and Trees.

LEON HART.

76 Brickyards on the Hudson. 
WALTER PACH.

77 Portrait.

BERNARD GUSSOW.

78 Landscape.

ARTHUR B. DAVIES.

79 Strong Toil of Grace.

GEORGE F. OF.

80 Portrait.

BERNARD GUSSOW.

8I Trees.

GUS MAGER.

82 Maine Pool.

HUGO ROBUS.

83 The Concrete Mixer.

J. TORRES-GARCIA.

84 Fashion.

WOOD GAYLOR.

85 Edna.

ARTHUR B. CARLES.

86 Monotype.

VINCENT CANADÉ.

87 Still Life. 
LOUIS BOUCHÉ.

88 East and West.

HOMER BOSS.

89 The Dawn.

JUSTUS PFEIFFEN.

90 Figures.

JOHN SLOAN.

9I Girl with Flowers.

WM. ZORACH.

92 Adoration.

C. BERTRAM HARTMAN.

93 Moon Path.

VINCENT CANADÉ

94 Landscape.

WILLIAM YARROW.

95 Flowers.

Lent by Mr. Maurice S. Speiser.

CHARLES DEMUTH. 96 Business.

VINCENT CANADÉ.

97 Little Trees.

MARGUERITE ZORACH.

98 Camp in the Woods. 
JENNINGS TOFEL.

99 Ascending.

GEORGE CANADÉ. Ioo Landscape.

ANDREW DASBURG. Ior Landscape.

VINCENT CANADÉ. 102 Landscape.

JUSTUS PFEIFFEN. I03 The Park.

MARION H. BECKETT. I04 Wyomissing Iris.

KONRAD CRAMER. I05 Man Reading.

JOHN PANDICK. 106 Still Life.

SAMUEL HALPERT.

ro7 Village Near the Sea.

JOHN SLOAN. 108 Etching. rog Etching. 


\section{PAINTINGS AND DRAWINGS.}

MACDONALD WRIGHT.

rro Painting: Number One.

Lent by Mr. Maurice J. Speiser.

JOHN SLOAN.

III Etching.

II2 Etching.

PRESTON DICKINSON.

II3 Landscape: Number Two.

ANDREW DASBURG.

II4 Village Street.

ALFRED J. FRUEH.

II5 Drawing.

SARA CARLES.

II6 Study.

SARAH LANGLEY.

Ir7 Improvisations: Number Two.

MARION H. BECKETT.

II8 Tiger Lilies.

FLORA SCHOENFELD.

II9 Mountain Home: Santa Fé.

JEAN KNOX.

I20 Still Life. 
BEN BENN.

I2I Landscape.

GEORGE F. OF.

I22 Landscape.

ARTHUR G. DOVE. I23 A Walk: Poplars.

Lent by Mr. Charles Daniel.

D. W. MCCOUCH.

I24 Lake Maggiore.

ARTHUR G. DOVE.

I25 League of Nations: rgr4.

Lent by Mr. Charles Daniel.

ANDREW DASBURG.

I26 Landscape.

JENNINGS TOFEL.

I27 Street with Mountain. 


\section{GALLERY G.}

THOMAS H. BENTON.

I28 Landscape.

GASTON LACHAISE.

I29 Drawing.

ALFRED J. FRUEH.

I30 Drawing.

C. BERTRAM HARTMAN.

I3I Village: Stonington, Maine.

PRESTON DICKINSON.

$r_{32}$ House Forms and Hills.

ARTHUR B. DAVIES.

I33 Tragic Mask.

EDWARD FISK.

I34 Mountain Lake.

JAMES H. DAUGHERTY.

I35 The Hands of Moses.

ALFRED J. FRUEH.

I36 Drawing. 
BEN BENN.

I37 Landscape.

MARGUERITE ZORACH.

I38 Camp at Night.

KONRAD CRAMER.

I39 Under the Tent.

CARL KAHLER.

I40 A Negro Head.

GEORGIA O'KEEFFE.

I4I Red.

142 Pink.

I43 Black Spot.

MORRIS KANTOR.

I44 Head.

CARL KAHLER.

I45 A Barn.

WM. ZORACK.

I46 Yosemite Landscape.

WILLIAM YARROW.

I47 Man in Action.

LEON HART.

I48 Germinal. 
HORACE BRODZKY.

I49 Water Color.

LEON HART.

I50 Fructidor.

MARGUERITE ZORACH.

I5I Yosemite Trails.

ARTHUR B. DAVIES.

I52 Three Masks.

C. BERTRAM HARTMAN.

I53 Evening Light.

CARL KAHLER.

I54 Creation.

A. WALKOWITZ.

I55 Drawing.

HORACE BRODZKY.

I56 Water Color.

I57 Peasants.

I58 New Rochelle.

JOHN COVERT.

I59 Resurrection.

HENRY L. MCFEE, I60 Still Life. 
JOHN MARIN.

I6I Weehawken

I62 Woolworth Building.

I63 The Brooklyn Bridge and City.

r64 Brooklyn Bridge.

JOHN COVERT.

I65 Temptation of St. Anthony.

THOMAS H. BENTON.

I66 Study for a Decoration.

A. H. MAURER.

I67 Head: Number Four.

JULES PASCIN.

I68 Street Corner in New Orleans.

169 Street in Havana.

Lent by Mr. Ferdinand Howald.

I7o In a Park.

Lent by Mr. Ferdinand Howald.

I7I Conversation.

A. H. MAURER.

I72 Head: Number Three.

CHARLES DEMUTH.

I73 In Vaudeville.

Lent by Mr. Charles Daniel.

OWEN MERTON.

I74 Hillside.

JOSEPH STELLA.

I75 Silverpoint: Ist.

I76 Silverpoint: 2 nd. 


\section{OWEN MERTON.}

\section{I77 Coming Storm.}

I78 Marseilles.

HENRY MCCARTER.

I79 House of Worship.

WOOD GAYLOR.

I80 Drawing.

OWEN MERTON.

I8I View of Flushing.

WOOD GAYLOR.

I82 Drawing.

WILLIAM YARROW.

I83 Rocks.

CHARLES DEMUTH.

I84 Class 5 G.

OWEN MERTOH.

I85 The Village.

JOHN MARIN.

I86 Moving Showers.

I87 Rocks and Sea: Maine.

Lent by Mr. Alfred Stieglitz.

I88 Moonlight.

I89 Mountain Shapes and Sky.

I90 Low Tide: Moose Island, Maine.

Lent by Mr. Alfred Stieglitz. 
PAINTINGS AND DRAWINGS.

JOSEPH STELLA.

IgI Flowers.

EDWARD FISK.

Ig2 The Mountain.

PAUL BURLIN.

I93 Concentric Energy.

I94 Drawing.

EDWARD FISK.

I95 The Farm. 


\section{NORTH TRANSEPT.}

GASTON LACHAISE.

I96 Drawing.

I97 Drawing.

MAURICE STERNE.

I98 Elizabeth Duncan: Dancer.

GASTON LACHAISE.

I99 Drawing.

200 Drawing.

WILLIAM YARROW. 20I Flowers.

CHARLES SHEELER. 202 Still Life: Drawing.

MAURICE STERNE. 203 Seated Figure.

A. WALKOWITZ. 204 Drawing.

ANDREW DASBURG. 205 Drawing. 
PAUL BURLIN. 206 Small Orchestra.

ANDREW DASBURG. 207 Drawing.

MARIUS DE ZAYAS. 208 Darnton.

Lent by Mr. Alfred Stieglitz.

\section{CHARLES SHEELER.} 209 Bucks County Barn.

GUS MAGER. 2 Io Farm Idyl.

MARIUS DE ZAYAS.

2 II John Marin and Alfred Stieglitz. Lent by Mr. Alfred Stieglitz.

MAURICE STERNE.

212 Taos Indian Woman.

\section{J. PASCIN.}

2I3 Etching.

Lent by Mr. Wood Gaylor.

2 I4 Cuban Lovers.

Lent by Mr. Wood Gaylor.

2 I5 Etching.

Lent by Mr. Wood Gaylor.

2I6 Etching.

Lent by Mr. Wood Gaylor.

ALFREQ J.ERUEH.

217 Drawing. 
MAURICE STERNE. 218 Dancer in Action.

MARIUS DE ZAYAS.

219 Katharine N. Rhoades.

Lent by Mr. Alfred Stieglitz.

ALFRED J. FRUEH.

220 Drawing.

MARIUS DE ZAYAS.

22 Marion Beckett.

Lent by Mr. Alfred Stieglitz.

ROBERT LAURENT. 222 Rocks.

LOUIS BOUCHÉ. 223 English Style.

KONRAD CRAMER. 224 Girl Dressing.

RAFAEL SALA. 225 A Beach of Catalonia.

BEN BENN.

226 Study of a Woman's Head.

E. B. GROSSMAN.

227 Nude Study.

228 Nude Model.

A. WALKOWITZ. 229 New York. 


\section{GALLERY F.}

MORTON L. SCHAMBERG.

230 Painting: Number One.

JOHN COVERT.

23I Brass Band.

WALTER PACH.

232 Sunday Night.

MARSDEN HARTLEY.

233 Still Life: Black Leaves.

WALT KUHN.

234 The City.

S. MACDONALD WRIGHT.

235 Aeroplane: Synchromy in Yellow and Orange.

EMILE BRANCHARD.

${ }_{23} 6$ Old Chestnuts.

LOUIS BOUCHÉ.

237 Portrait: Horace Brodzky.

MAX WEBER.

238 Portrait. 
EMILE BRANCHARD.

239 Birch Trees.

PRESTON DICKINSON.

240 Landscape: Number One.

A. S. BAYLINSON.

$24 \mathrm{I}$ Figure.

ARTHUR B. DAVIES.

242 On Violence.

243 Mænad Arabesque.

JOSEPH STELLA.

244 Battle of Light: Coney Island.

MAN RAY.

245 MCMXIV.

MAURICE STERNE. 246 Flower in Vase.

BERNARD GUSSOW.

247 Passing Figures.

LOUIS BOUCHÉ. 248 Still Life.

HOMER BOSS.

249 The Red Fog. 
JOHN MARIN.

250 Tree Form.

S. MACDONALD WRIGHT.

25 I Synchromy.

Lent by Mr. Jacob Dewald.

EDWARD J. STEICHEN.

252 Mural Decoration: Fragment.

Lent by Mr. Eugene Meyer, Jr.

MARSDEN HARTLEY.

253 Flowers in Glass.

MAX WEBER.

254 Chinese Restaurant.

MARSDEN HARTLEY.

255 Landscape: New Mexico.

THOMAS H. BENTON.

256 The Beach.

MARSDEN HARTLEY.

257 Movement: Provincetown.

EDWARD J. STEICHEN.

258 Mural Decoration: Fragment.

Lent by Mr. Eugene Meyer, Jr.

CHARLES SHEELER.

259 Bucks County Barn.

JOSEPH M. GARVEY.

260 The Masked Ball. 
WM. ZORACH. 261 Ships Passing at Night.

CHARLES SHEELER. 262 New York.

LOUIS BOUCHÉ. 263 Still Life.

WM. ZORACH. 264 Interior and Exterior.

WILLIAM YARROW. 265 Shadow-Boxer.

D. W. MCCOUCH. 266 Village Entrance.

ARTHUR B. DA VIES. 267 Façade.

GEORGE F. OF. 268 The Church.

MAN RAY.

269 Departure of Summer.

CHARLES DEMUTH.

270 Aucassin and Nicolette. Lent by Mrs. Meredith Hare.

PAUL BURLIN.

27I Awakening. Lent by Mr. George A. Harris. 28 


\section{PAINTINGS AND DRAWINGS.}

\section{CHARLES DEMUTH.}

272 From the Garden of the Château.

HENRY L. MCFEE.

273 Portrait of a Painter.

CARL KAHLER.

274 A Mechanism.

ARTHUR B. CARLES.

275 Profile.

MORTON L. SCHAMBERG.

276 Painting: Number Two.

WALT KUHN.

277 Youth.

Lent by Mr. John Quinn.

JOSEPH STELLA.

278 Tropical Sonata.

WOOD GAYLOR.

279 Posters.

MORTON L. SCHAMBERG. 280 Painting: Number Three. 


\section{INDEX TO ARTISTS}

REPRESENTED IN THE PRESENT EXHIBITION.

The figures following the address refer to the numbers in this catalogue.

Baylinson, A. S.,

I947 Broadway, New York City, 4r, 60, 24I.

Beckett, Marion H.,

30 East Sixtieth Street, New York City,

Benn, Ben, I04, I18.

244 East Twenty-third Street, New York

Benton, Thomas H.,

City, 39, I2I, I37, 226.

402 East Thirty-second Street, New York

Biddle, George,

City, I 28, I66, 256.

52 East Fifty-third Street, New York City,

Boix, Richard,

$$
\text { I6, 20, 32, } 48 \text {. }
$$

49 West Eighth Street, New York City, 27,30 .

Boss, Homer,

37 West Sixteenth Street, New York City,

Bouché, Louis,

$89,249$.

The Daniel Galleries, 2 West Forty-seventh

Street, New York City, 88, 223, 237, 248, 263

Branchard, Emile,

6I Washington Square, New York City, I7,

Brodzky, Horace,

$$
\text { 2I, 64, 65, 66, 236, } 239 .
$$

I4I East Twenty-seventh Street, New York

Burlin, Paul,

City, I 49, I 56,157, I 58.

I06 West Fifty-seventh Street, New York

City, I93, 194, 206, 27 I. 
Canadé, George,

I454 Sixty-fifth Street, Brooklyn, New

Canadé, Vincent, York, Ioo. I454 Sixty-fifth Street, Brooklyn, New York,

Carles, Arthur B., $87,94,97$, I02. 201 South Tenth Street, Philadelphia, 86,

Carles, Sara, 275 .

2007 Girard Avenue, Philadelphia, i 16.

Covert, John,

I5 West Twenty-ninth Street, New York

Cramer, Konrad,

City, I59, I65, 231.

Woodstock, U1ster County, New York, 105.

Cummings, E. E.,

I39, 224

I04 Irving Street, Cambridge, Massachu-

Dasburg, Andrew, setts, 3I, 62 .

72 Washington Square, New York City,

Daugherty, James H., IOI, II4, I26, 205, 207.

59 South Washington Square, New York

Davies, Arthur B.,

City, I3, 22, I35.

337 East Fifty-seventh Street, New York

City, 79, I33, I52, 242, 243, 267.

Diederich, Hunt,

$50 \frac{1}{2}$ Barrow Street, New York City, 52, 55.

Demuth, Charles,

2 West Forty-seventh Street, New York

De Zayas, Marius,

City, 96, 173, 184, 270, 272 .

549 Fifth Avenue, New York City, 208, 2 II, 2I9, 22 I. 
Dickinson, Preston,

The Daniel Galleries, 2 West Forty-seventh Street, New York City, I0, II3, I32, 240.

Dove, Arthur G.,

Westport, Connecticut, I23, 125.

Dreier, Katherine L.,

In care of George Of, 274 Madison Avenue,

Field, Hamilton Easter,

New York City, 33. Io6 Columbia Heights, Brooklyn, New

Fisk, Edward, York, 36.

The Daniel Galleries, 2 West Forty-seventh Street, New York City, 9, I5, I34, I92, 195.

Friedman, Arnold

53 Baylies Street, New York City, 37, 43.

Frueh, Alfred J.,

34 Perry Street, New York City, I I5, I30,

Garcia, J. Torres-

I36, 2े $7,220$.

4 West Twenty-ninth Street, New York

Garvey, Joseph M.

City, 4, 24, 44, 84 .

Alpine, New Jersey, 5, 260.

Gaylor, Wood,

I42 West One Hundred and Twenty-seventh Street, New York City, 59, 85, I80, I82, 279.

Glackens, William,

Io West Ninth Street, New York City, 68,70 .

Grossman, E. B.,

133 East Twenty-first Street, New York

Gussow, Bernard,

City, 3, 227, 228.

54 Charles Street, New York City, 46, 78, $8 \mathrm{r}, 247$. 
Halpert, Samuel,

I28 West Eighty-fifth Street, New York

Hart, Leon,

City, I2, 107.

3II West Twenty-fourth Street, New York

Hartley, Marsden,

City, 76 , 148, I 50

The Daniel Galleries, 2 West Forty-seventh Street, New York City, 233, 253, 255, 257.

Hartman, C. Bertram,

36 Perry Street, New York City, 2, 93, I3I,

Kahler, Carl

I 53 .

- 49 West Eighth Street, New York City, I40,

Kantor, Morris, I 45, I 54,274 .

I947 Broadway, New York City, 26, 42, I44.

Knox, Jean,

II5 Carpenter Lane, Mount Airy, Philadel-

Kuhn, Walt, phia, I20.

II East Thirteenth Street, New York City,

Kuniyoshi, Yasuo, $234,277$.

I IO Columbia Heights, Brooklyn, New

Lachaise, Gaston, York, 54

77 Washington Place, New York City, I29,

Langley, Sarah, 196, 197, 199, 200.

218 Narberth Avenue, Narberth, Pennsylvania, II7.

Laurent, Robert,

Io6 Columbia Heights, Brooklyn, New

McCarter, Henry,

York, 7I, 75, 222.

200 South Fifteenth Street, Philadelphia,

I79. 
McCouch, D. W.,

McFee, Henry L.,

St. Martins Lane, Chestnut Hill, Philadelphia, 6, 124, 266.

The Daniel Galleries, 2 West Forty-seventh Street, New York City, I6o, 273.

Mager, Gus,

So Glen Avenue, Wyoming, New Jersey, 74,

Marin, John,

82,210 .

Cliffside, Bergen County, New Jersey, I6I, I62, 163, I64, I86, I87, I88, I89, 190, 250.

Maurer, A. H.,

404 West Forty-third Street, New York

Merton, Owen,

City, 72, 167, I72.

57 Hillside Avenue, Flushing, Long Island,

New York, I74, 177, I78, I81, 185.

Nagel, Elizabeth,

77 Washington Place, New York City,

Nagle, Edward,

$34,38,63$.

77 Washington Place, New York City, 19.

Newman, Carl,

Beth Ayres, Pennsylvania, 49, 50, 5 I.

Nordfeldt, B. J. O.,

Santa Fé, New Mexico, 7, 57.

Of, George $F_{\text {, }}$

2794 Morris Avenue, New York City, 23,

O'Keeffe, Georgia,

80, 122, 268 .

6o East Sixty-fifth Street, New York City,

Pach, Walter,

I4I, I42, I43.

I3 East Fourteenth Street, New York City, $28,77,232$. 
Pandick, John,

Fanwood, New Jersey, II, I4, 106.

Pascin, Jules,

The Daniel Galleries, 2 West Forty-seventh Street, New York City, I68, I69, I70, I7I, $213,214,215,216$.

Pfeiffen, Justus,

In Care of Preston Dickinson, Valley Stream, Long Island, New York City, 90,

Ray, Man,

I03.

47 West Eighth Street, New York City,

Robus, Hugo,

245, 269 .

9 East 14th Street, New York City, I8, 47,

Sala, Rafael,

216 West Fiftieth Street, New York City, $25,69,225$.

Sayen, Lyman,

Deceased, 56, 58 .

Schamberg, Morton L.,

In Care of W. Pach, I3 East Fourteenth Street, New York City, 230, 276, 280.

Schoenfeld, Flora,

5024 Ellis Avenue, Chicago, Illinois, Irg.

Sheeler, Charles,

In Care of M. de Zayas, 549 Fifth Avenue, New York City, 202, 209, 259, 262.

Sloan, John,

88 Washington Place, New York City, 9r, 108, 109, III, II2.

Steichen, Edward J.,

I2I West Sixty-eighth Street, New York City, 252, 258. 


\section{INDEX.}

Stella, Joseph,

213 West Fourteenth Street, New York City, 35, 175, 176, 191, 244, 278.

Sterne, Maurice,

668 Fifth Avenue, New York City, 53, I98,

Stettheimer, Florine, 203, 212, $218,246$.

80 West Fortieth Street, New York City,

Taylor, Henry Fitch, I, 29.

Cos Cob, Connecticut, 40, 61 .

Tofel, Jennings,

6I Colden Street, Newburgh, New York,

Tucker, Allen, $99,127$.

I2I East Seventy-ninth Street, New York

Walkowitz, A., City, 8, 67 .

I2 Union Square, New York City, I55, 204, 229.

Watson, Nan,

I2 West Eighth Street, New York City,

Weber, Max,

66 Post Avenue, New York City, 238, 254

Wright, S. MacDonald,

Los Angeles, California, I I0, 235, 25 r.

Yarrow, William,

26 East 62nd Street, New York City, 95, $147,183,201,265$.

Zorach, Marguerite,

I23 West Tenth Street, New York City,

Zorach, William, I38, $15 \mathrm{I}$.

123 West Tenth Street, New York City, 45, $92,98,146,26 \mathrm{r}, 264$. 
$6 \%$ and 





$$
\text { - }
$$


SMITHSONIAN INSTITUTION LIBRARIES

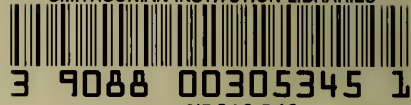

nmaa ND212.P42

Paintings and drawings by American artis 